\title{
Cataract extraction without retrobulbar anaesthetic injection
}

\author{
Redmond Smith
}

\begin{abstract}
Extracapsular cataract extraction with lens implant under local anaesthesia consisting of amethocaine drops followed by a simple subconjunctival injection in the upper part of the globe but without a retrobulbar injection was carried out in 175 eyes of 165 patients. The purpose of the study was to establish the feasibility of this type of anaesthesia in cataract surgery with the principal object of avoiding the possibility of retrobulbar haemorrhage and the other, rarer, complications of retrobulbar injection. The method, which is referred to as the NR method, proved to be satisfactory. No undue difficulties were encountered as a result of using the technique, the visual results were satisfactory and compared favourably with those under general anaesthesia, and the patients accepted the method well, possibly preferring it to conventional methods.
\end{abstract}

Local anaesthesia for cataract has usually included a retrobulbar injection of a solution such as xylocaine or one of the longer acting anaesthetics. The reason for taking what to the casual observer seems a somewhat alarming step, namely, the plunging of a long and extremely sharp needle some 4 to $5 \mathrm{~cm}$ into the orbit in close proximity to the globe, has been the supposition that anything less would be likely to give less than adequate anaesthesia and akinesia. As has been pointed out recently, however, retrobulbar anaesthesia is not so essential a step as is widely believed. ${ }^{1}$ Furthermore it is not without complications. Accidental perforation of the eye ${ }^{2}$ transitory blindness, ${ }^{3}$ central retinal artery occlusion, ${ }^{4}$ and cranial nerve palsy ${ }^{5}$ have all been reported.

The present study arose out of a fortuitous occurrence. In March 1985 two successive cataract extractions had to be postponed owing to retrobulbar haemorrhage, and although, fortunately, no serious complications ensued, nevertheless the possibility of doing without the retrobulbar injection was considered. Simple peripheral iridectomy for angle closure glaucoma and fistulising operations of various sorts for open-angle glaucoma had always been performed without retrobulbar injection in the author's practice, but for the reasons mentioned above there had been no previous attempt to use the technique for cataract surgery. A trial of cataract extraction without retrobulbar anaesthesia was therefore started in April 1985 and continued until April 1988. Material and methods As time was relatively short owing to impending retirement it was decided to carry out a simple open trial of the technique, in effect a feasibility study rather than a comparative trial with formal controls. All patients listed for cataract extraction under local anaesthesia were (with a few exceptions to be mentioned later) operated on by the no retrobulbar (NR) technique. Approximately half of these were carried out at the Western Ophthalmic Hospital, London, and the remainder at a private clinic. All operations were performed by the author.

The total number of extractions during the period was 208 in 193 patients; 118 were female and 75 male. The mean age was 70.5 years (range 46-90, SD 14.5). The apparent infrequency of bilateral cases was due to two principal reasons. First, the operation on the other eye occurred outside the trial period; or, secondly, the other eye was operated upon by another surgeon in the clinical firm.

The total number of local anaesthetic (LA) operations in the series was 178 , of which three were carried out by a retrobulbar and 175 by the NR technique. Thirty eyes were operated upon under general anaesthesia (GA). In one patient a general anaesthetic was used for the first eye and a local for the second. There were no patients in whom a local was used in the first eye and a general in the second. LA was used on both eyes of nine patients and GA on both eyes of four patients.

One patient operated on by the NR technique had simultaneous squint surgery.

\section{NR TECHNIQUE}

The NR technique was carried out as follows. No premedication was used. Patients were reassured that little or no pain was likely to be felt. Some patients were afraid that they might prejudice the result of the operation by inadvertent movements but were reassured that this was in our experience extremely unlikely. Before towelling up the head two drops of amethocaine $1 \%$ were instilled into the eye, care being taken to apply the drops to the upper bulbar conjunctiva. Two or three minutes later a subconjunctival injection of up to $1 \mathrm{ml}$ of $2 \%$ xylocaine was made in the upper bulbar region about $7 \mathrm{~mm}$ from the limbus. No attempt was made to enter the belly of the superior rectus muscle for fear of accidentally perforating the globe and the needle was always 'agitated' slightly from side to side to make certain that it was indeed in loose subconjunctival tissue only, before the injection was actually made. The bleb resulting from the injection was massaged downwards on the globe and could always be seen to extend to below the 3 and 9 o'clock positions on the limbus. In a few of 
over the medial and lateral recti, but this was found to be unnecessary and was therefore not persisted with. Facial block by O'Brien's technique was given up to June 1987 but was then discontinued. A Clark's speculum was used to hold the eye open during the surgery. This particular speculum, though it looks old fashioned, is extremely efficient and has two advantages over specula depending on a spring action to keep the eye open. First, the amount of opening is totally under the control of the surgeon, so that unduly forceful opening of the lids is avoided. Secondly, the speculum, once locked open at the separation chosen by the surgeon, is not subject to forced closure by the patient 'squeezing' - hence the reason for being able to dispense with facial block. The final manoeuvre before starting the extraction was the insertion of a superior rectus suture. Extraction was carried out by the extracapsular technique with a sequence of can-opener capsulotomy, simple expression, and cortical removal with a McIntire or similar canula. All the eyes had an intraocular lens implanted - a Sinskey type lens in most but a Severin in one and an unspecified lens in another. These were two eyes with vitreous loss. A third eye with vitreous loss could be seen to have only a limited hole in the posterior capsule and had a Sinskey lens with satisfactory results.

\section{Results}

Results may be considered from three aspects: the visual results of the operations, the difficulties and complications encountered, and the patient's responses to the technique.

VISUAL RESULTS

With such small numbers subtle differences from the expected norms would not be likely to show up, and, as mentioned in the introduction, this trial was no more than a feasibility study. However, purely for illustrative purposes, with no pretensions to statistical significance, the visual results of the three types of anaesthesia used during the period - NR, conventional retrobulbar, and GA - are displayed in Table I.

It can be seen from Table I that the visual results expressed as the visual acuities at approximately three months after operation appear reasonably satisfactory and there is no obvious difference between the NR and GA cases. Furthermore there is certainly no obvious difference between these results and what one has come to expect from local anaesthesia by conventional retrobulbar injection.

DIFFICULTIES AND COMPLICATIONS

The expected difficulties due to excessive eye

TABLE I Visual results of three types of anaesthesia

\begin{tabular}{|c|c|c|c|c|c|}
\hline \multirow[t]{2}{*}{ Anaesthesia } & \multicolumn{5}{|c|}{ Visual acuity } \\
\hline & $6 / 5-6 / 6$ & $6 / 9-6 / 18$ & $6 / 24-6 / 60$ & Less than $6 / 60$ & No record \\
\hline $\begin{array}{l}\text { NR } \\
\text { Retro } \\
\text { GA }\end{array}$ & $\begin{array}{c}54(31 \%) \\
1- \\
10(33 \%)\end{array}$ & $\begin{array}{c}101(59 \%) \\
0- \\
16(54 \%)\end{array}$ & $\begin{array}{l}9(5 \%) \\
1- \\
3(5 \%)\end{array}$ & $\begin{array}{l}8(4 \cdot 5 \%) \\
0- \\
1(3 \%)\end{array}$ & $\begin{array}{l}3(1 \cdot 5 \%) \\
1- \\
0-\end{array}$ \\
\hline
\end{tabular}

movements did not materialise. Moderate eye movements occurred in a few patients, but the impression gained was that the movements were no more troublesome than those which occur at times in retrobulbar cases, and there were no cases in which eye movements made any substantial difference to the ease with which the operation could be carried out. One patient made an alarming convulsive head movement during the operation, but this could have equally well happened during retrobulbar anaesthesia. Vitreous loss occurred in three eyes during NR. There was no vitreous loss in the GA cases. All the vitreous loss cases had a lens implanted, as mentioned previously, and the final visual acuities were $6 / 6,6 / 9$, and $6 / 9$ respectively in the three eyes. Two other patients had had vitreous loss in their other eyes previous to the study while being operated upon under retrobulbar anaesthesia, one by me and one by a resident. The two patients whose retrobulbar haemorrhages in March 1985 had prompted the initiation of the study were eventually operated on during the study uneventfully by the NR technique.

\section{PATIENTS' RESPONSES}

Many patients were surprised at the painlessness of the procedure. The impression gained was that, owing to the preliminary conjunctival surface anaesthetic and the relatively shallow depth of the subconjunctival injection, the average patient actually did not feel any injection being given at all, and this effect was of course even more striking in those patients who did not have a facial block. Contrary again to what one had been led to expect (though in accord with experience during glaucoma surgery) those few patients who had an iridectomy rarely seemed to feel pain. Most patients, however, experienced some discomfort during the insertion of the conjunctival sutures into the upper lip of the wound at the conclusion of the operation. But in my experience this is exactly the same as what happens after retrobulbar anaesthesia, though it may be better after a long-acting type of local anaesthetic, material with which I have had no personal experience.

Only one patient experienced severe pain during the operation, and it has to be admitted that this was a distinctly unnerving experience. The patient was a very obese West Indian female on whom the local anaesthetic seemed to be almost ineffective. As she was adamantly opposed to GA, the second eye was operated on with a retrobulbar anaesthetic, and I was surprised to find that this proved to be even worse than the NR operation. Fortunately both procedures were carried out satisfactorily, but it seemed clear that in this particular patient it was not the NR technique which had caused her pain in the first operation but a resistance to the anaesthetic material itself.

None of the patients operated on by the NR technique requested a different technique for the second eye.

\section{Discussion}

One of the most obvious differences between 
surgeons and even institutions is the large variations that occur in the ratio between numbers of cataracts done under LA and under GA. It is unlikely that the populations being operated on differ substantially from one another, especially within one country, so that the indications for LA or GA must depend more on the personal preferences of the surgeons than the medical condition of the patients. One is told repeatedly that old and infirm patients are just as safe under GA as LA, but I have to confess that I personally doubt whether this is always true. Furthermore, from a purely financial point of view, if extensive day-case surgery is going to become more widely practised, skill and confidence in local anaesthesia need to be improved. I have the distinct impression that there are some surgeons, of the highest skill in many cases, who are distinctly uncomfortable with local anaesthesia. This probably accounts for the high GA/LA ratio in some centres. The difference between conven- tional retrobulbar LA and the NR technique is not great; it is in fact a difference of detail only. Surgeons will have to judge for themselves whether the fact that the NR technique appears to be, if anything, pleasanter for patients than retrobulbar anaesthesia, and that it avoids the possibility of retrobulbar haemorrhage, not to mention the other admittedly rare complications, is sufficient reason for their abandoning retrobulbar anaesthesia and trying the NR technique.

1 Smith RJH. Why retrobulbar anaesthesia? Br $\mathcal{f}$ Ophthalmol 1988; 72 : 1 .

2 Lincoff H, Kreissig I. Local anaesthetic with accidental perforation of the eye. Klin Monatsbl Augenheilld 1986; 188: foration

3 Marx P, Langois J, Brasseur G. Transitory blindness after retrobulbar anaesthesia. Bull Soc Ophthalmol Fr 1979; 79: 925-8.

4 Klein ML, Jampol LM, Condon PI, Rice TA, Serjeant GR. Central retinal artery occlusion without retrobulbar haemorrhage after retrobulbar anaesthesia. Am J Ophthalmol 1982; 93: 573-7.

5 Rodgers R, Orellana J. Cranial nerve palsy following retrobulbar anaesthesia. Brf Ophthalmol 1988; 72: 78. 